\title{
La traducción de la Arcadia de Sannazaro por Jerónimo Jiménez de Urrea. Estudio y edición crítica de la égloga XII
}

\author{
Cecilia Cañas Gallart \\ Universitat de Barcelona \\ ccanas2@gmail.com
}

Recepción: 09/07/2014, Aceptación: 18/07/2014, Publicación: 17/12/2014

\begin{abstract}
Resumen
La fortuna de la Arcadia de Sannazaro en la Espańa del siglo xvi estuvo precedida y acompañada por traducciones cuyo análisis ofrece interesantes datos sobre la recepción de que fue objeto. Tras la adaptación parcial de la Prosa viII por Garcilaso en su Égloga II, y la primera traducción completa de Diego López de Ayala y Diego de Salazar, editada en 1547, otros tres literatos — Juan Sedeńo, Jerónimo de Urrea, y un Licenciado Viana - prepararon versiones ex novo de las églogas, todas ellas inéditas a día de hoy. En este artículo se publica por primera vez, en edición crítica, un extracto de la realizada por Urrea. Para ello se ha elegido la Égloga XII: la más extensa y ambiciosa, que compendia el sentido de la obra. Se describe asimismo el manuscrito, cuyas licencias y permisos avalan un proceso claramente encaminado a la impresión del texto.
\end{abstract}

Palabras clave

Jerónimo Jiménez de Urrea; Arcadia; Sannazaro; traducción; égloga XII

\begin{abstract}
XVI th.c. Jerónimo Jiménez de Urrea's translation of Sannazaro's "Arcadia", an essay and a critical edition of XIIth eclogue

Sannazaro's Arcadia's success in XVI century Spain was preceded by and coexisted with other translations. Their analysis offers remarkably interesting data about the Arcadia's reception, recognition and acceptance at the time. After a partial adaptation of Prose VIII by Garcilaso in his Eclogue II, and Diego López de Ayala’s and Diego de Salazar's first complete translation published in 1547, there were three other ex novo versions, the ones by Jerónimo de Urrea, Juan Sedeño and Licenciado Viana, which still remain unpublished
\end{abstract}


nowadays. An extract of Jerónimo de Urrea's translation is published, for the first time in a critical edition, in this essay. In order to accomplish it we have chosen the Eclogue XII, which is the most ambitious and thorough. Eclogue XII also embodies and summarises the sense and significance of Sannazaros's work. We depict his manuscript whose permits and licenses guarantee and confirm a process clearly aimed at text printing.

Keywords

Jerónimo Jiménez de Urrea; Arcadia; Sannazaro; translation; eclogue XII

\section{Premisa}

La fortuna de la Arcadia comenzó muy pronto en Italia, como muestra el alto número de ediciones que tuvo ya en la primera mitad del xvi; luego, siguiendo un proceso distinto en cada país, se convirtió en un modelo imitado ampliamente en Europa durante ese siglo y el siguiente. A esta difusión contribuyeron las traducciones: la primera aparecida en prensas fue la francesa, realizada por Jean Martin en 1544, aunque se adelantó solo en tres años a la española de López de Ayala y Diego de Salazar, editada en 1547 por las prensas toledanas de Juan de Ayala y al cuidado de Blasco de Garay. Sin embargo, el primer ejemplo de la huella sannazariana en Europa lo constituye Garcilaso de la Vega, con la temprana adaptación versificada de un extenso fragmento de la prosa viII en su Égloga II (vv. 161-680). ${ }^{1}$ No hace falta resaltar la importancia de este primer eslabón para el arraigo de la bucólica lírica en castellano y portugués, pero si ello ha ocupado desde antiguo a la crítica, escasean, en cambio, los estudios sobre las traducciones propiamente dichas, ${ }^{2}$ y en particular sobre tres versiones

1. Para un análisis detallado de la trasposición métrica de la sintaxis sannazariana por parte de Garcilaso, cf. Gargano (2007): 347-359. Rafael Lapesa observa que Sannazaro «fue el autor de la actualidad italiana que más influyó sobre Garcilaso», Lapesa (1968): 93. Para su datación, remitimos a Bienvenido Morros: «La égloga II suele fecharse en los primeros meses de la estancia del poeta en Nápoles y pudo haberse compuesto en distintos momentos con poca distancia temporal entre sí (mayo de 1533 y principios de 1534)», Morros (2001): 147.

2. Con una sola excepción, la de Rogelio Reyes Cano, cuya tesis, publicada en 1973, reeditó la edición de 1547 y abordó por vez primera, aunque de forma muy somera, el examen de las versiones inéditas ofreciendo algunas noticias sobre sus autores y esbozando un cotejo de sus resultados métricos y estilísticos aunque su centro siguió siendo la edición de Garay. Cf. Reyes Cano (1973). 
quinientistas inéditas, cuyo análisis no puede soslayarse para comprender plenamente el alcance de la recepción de la obra. Nuestro trabajo se propone romper esta inercia, editando por primera vez, aunque sea de modo parcial, el texto de Jerónimo Jiménez de Urrea. Intentamos así preservar y valorizar una pieza no secundaria del patrimonio histórico-literario del Siglo de Oro, que añade nuevos datos tanto para la historia de las traducciones renacentistas, como para el conocimiento cabal de la obra de Urrea. Nuestra selección ha recaído en la Égloga XII, que, además de ser la más extensa y de condensar el sentido principal de la obra, presenta un elevado grado de dificultad estructural y retórica, que ofrece gran riqueza de matices para comparar el método y la calidad de las distintas versiones.

De la única traducción impresa se conserva un manuscrito en la Biblioteca del Palacio Real, II/1331, que podría datarse entre 1535 y 1540. Diego López de Ayala vertió las prosas y Diego de Salazar, las églogas, aunque adaptándolas a diferentes formas estróficas de octosílabos, salvo 94 endecasílabos mantenidos en égloga x. El texto vio la luz solo en 1547 gracias a Blasco de Garay, racionero en la catedral de Toledo y autor de unas Cartas de refranes además de alguna poesía laudatoria. El éxito de esta traducción queda atestiguado por las cuatro ediciones que tuvo en menos de treinta años. En su prólogo, Garay calificaba a Diego López de Ayala «como fiel intérprete» de las prosas, y reiteraba su juicio favorable en la carta al lector situada al final del volumen. Bien distinto, en cambio, el valor atribuido a la traducción de los versos debida a Diego de Salazar, a quien reprochaba la excesiva libertad respecto a la letra original. Así, intervino con retoques puntuales de tipo léxico y semántico, aunque, en cuanto a la métrica, mantuvo la adaptación en coplas.

Volvamos, pues, a las traducciones inéditas. Todas ellas se conservan en la Biblioteca Nacional de Madrid: la de Urrea, con correcciones y sin fecha, se halla en un volumen manuscrito con la signatura Mss. 1469, que contiene también el poema épico inédito El Victorioso Carlos Quinto, con distintas aprobaciones y censuras que apuntan a su inminente impresión; la firmada por Juan Sedeño, figura como obra única en el códice Mss. 7716, con dedicatoria a Gonzalo Fernández de Córdoba, duque de Sessa, y sin aprobaciones; otro tanto cabe decir, en cuanto a la falta de aprobaciones o signos de futura impresión, de la tercera y última, firmada por un no identificado "Licenciado Viana», y cuyo manuscrito lleva la signatura Mss. 7486.

Ayuda a valorar en su justa medida la traducción urreiana recordar que se llevó a cabo en un período - la segunda mitad del s. Xvi — en el que la lengua castellana estaba evolucionando rápidamente mientras arreciaba el debate sobre su riqueza con respecto al latín y al italiano. No hace falta recordar cómo, en la traducción de El Cortesano, Boscán había llevado a la práctica la idea, compartida por Garcilaso, de naturalizar en castellano los textos vertidos, aunque respetando lo más posible la forma y estilo originales. En cuanto a la poesía, el afianzamiento del endecasílabo no podía no reflejarse en las traducciones, si 
bien el recurso de Salazar al metro castellano da la medida de un proceso todavía incierto en la época en la que se realizó la versión. Ya en el siglo siguiente, Cervantes mostraría en el Quijote hasta qué punto se había ido elevando el nivel de exigencia formal, al sostener que toda traducción de poesía comportaba una pérdida, aportando como prueba precisamente una traducción de Urrea, la del Orlando furioso, ${ }^{3}$ cuya distancia del original lamentaba al tiempo que reconocía la dificultad de la empresa. Esta valoración condicionaría la crítica venidera contribuyendo de modo decisivo a arraigar un prejuicio negativo sobre las versiones de Urrea. Cierto es que en 1866 Jerónimo Borao introdujo un criterio de relativización histórica que lo corregía, al considerar los límites de Urrea como efecto del estado de la lengua poética castellana y de las técnicas traductorias del tiempo (cf. Borao (1866): 7-56). Justo cien años después, Maxime Chevalier insistía en esta misma línea, mientras que Geneste hacía un balance en claroscuro en su biografía urreiana de $1974 .{ }^{4}$ Casi treinta años debían pasar aún para que Segre y Muñiz ofrecieran una edición bilingüe de su traducción de Ariosto que permitió cotejarla puntualmente con el original senáalando en notas críticas desviaciones y variantes (Orlando furioso (2002)). Ello permitió constatar la notable fidelidad de la versión y, pese a los consabidos desajustes formales, su innegable superioridad con respecto a otras coetáneas y posteriores (Ibid., vol. I, p. 42).

Volvamos, pues, a la Arcadia y a la decisión de Urrea de verterla al castellano.

Cuando en 1547 apareció la primera traducción impresa de la obra, el endecasílabo ya había arraigado en las letras españolas. Debió, pues, de resultar chocante que las églogas hubieran sido adaptadas a coplas castellanas cuando ya las Obras poéticas de Boscán y Garcilaso llevaban cuatro años circulando. El terreno estaba, en suma, abonado para poner en crisis cualquier traducción en verso que no respetara la estrofa original. Ello nos da la clave para explicar por qué tres distintos traductores decidieron emprender una nueva versión de las églogas sannazarianas. Se explica asimismo la menor atención prestada por todos ellos a la parte en prosa, para cuya traducción tomaron como punto de partida la versión impresa de López de Ayala, si bien aportando modificaciones de distinta entidad.

Pasemos, pues, a la traducción de Urrea, comenzando por describir el único manuscrito que la ha conservado.

3. «[...] y aquí le perdonáramos al señor capitán que no le hubiera traído a España y hecho castellano; que le quitó mucho de su natural valor, y lo mesmo harán todos aquellos que los libros de verso quisieren volver en otra lengua: que, por mucho cuidado que pongan y habilidad que muestren, jamás llegarán al punto que ellos tienen en su primer nacimiento» (I, 6).

4. «Partagé entre le désir de respecter le texte et celui de faire un vers juste, sollicité en sens contraire par ces deux propos, dépassé ou importuné par la tâche de les concilier, on le voit céder tantôt sur un article et tantôt sur l'autre, sacrifier tantôt la poésie à l'exactitude et tantôt l'exactitude à la versification», Geneste (1978): 208. 


\section{El manuscrito de la traducción de Urrea}

La traducción de la Arcadia forma parte de un volumen encuadernado en piel marrón sin tejuelo, con hierros dorados y cortes jaspeados del siglo XvII, que, como hemos dicho, guarda la Biblioteca Nacional de Madrid con la signatura Mss. 1469. El códice procede de la Biblioteca Real (Olim. M 224), y su encuadernación presenta las dimensiones 19’3 $315 \mathrm{~cm}$., mientras que los folios miden $18^{\prime} 8 \times 14^{\prime} 4 \mathrm{~cm}$. En primer lugar figura el poema El Victorioso Carlos Quinto; a continuación, la traducción de la Arcadia. Las dos obras están escritas a línea tirada, ocupando recto y verso. Su numeración — situada en el ángulo superior derecho, en caracteres arábigos y con tinta, presumiblemente coetáneos- es independiente: de 1 a 171 para El Victorioso Carlos Quinto, de 1 a 80 para la Arcadia. Hay, en fin, una pestaña pequeña de menos de un centímetro que separa ambos textos.

El volumen consta de 5 hojas de guarda $+(171+80+4$ hojas $=255)+2$ hojas de guarda, lo cual suma $262 \mathrm{~h}$. en total. De las 255 escritas, las primeras 249 están completas, mientras que las últimas 6 (del f. 250 al 255) aparecen mutiladas por un desgarro de la parte superior derecha que afecta a las tres páginas finales de texto de la Arcadia: exactamente las que van desde temendo egli di contristare le sue feste hasta el final, lo cual supone la pérdida en la traducción de dos tercios del epílogo $A$ la sampogna.

En la cuarta hoja figura la fecha 1584 en la parte superior derecha, y a continuación el título y la dedicatoria: $1584 \mid$ El Vitorioso carlos quinto | conpuesto pordongeronimo de $\mid$ Urrea dirigido ala S.C.R.Mo $\mid$ del Rey don Felipe n[ues]tro S.

En la hoja 5v, figura la licencia de edición del Victorioso Carlos Quinto con la firma de Alonso de Ercilla : «Eneste libro hallo muchas cosas buenas que son | la gravedad dela historia la grandeza y valor de | n[uest]ro príncipe ynación, el buen estilo verso y len | guage, con que se escrive por lo qual mepareçe | que es bien que se ymprima. Don Alonso de | ercilla y çuñiga». En el f. Gr inicia el texto del poema. Al final de este, en el f. 170v, consta una segunda autorización sin firma, en nombre del Arzobispo de Zaragoza, con fecha 5 de junio de 1579, mientras que el f. 171r está ocupado por una nueva autorización del mismo Arzobispado escrita con letra diferente, que lleva fecha de 15 de noviembre de ese mismo ańo, y la firma de Fray Francisco de Mansilla con su rúbrica al pie. Esta última rúbrica aparece asimismo al pie de todos y cada uno de los folios del ms., incluidos los correspondientes a la traducción de la Arcadia.

La traducción de la obra sannazariana ocupa 80 folios, de los cuales, como hemos dicho, se hallan mutilados los últimos, exactamente los que corresponden al recto y verso del f. 79 , y al recto del f. 80. Dada la amplitud del desgarro, no se

5. Cabe recordar a este respecto que el autor de la Araucana había firmado en 1583 la aprobación del Furioso de Urrea para la nueva estampa publicada en Bilbao (Orlando Fvrioso de M. Lvdovico Ariosto traduzido... por Don Geronymo de Vrrea, Bilbao, por Mathias Mares, 1583). 
puede excluir que el final del texto - f. 80r- contuviera alguna nota de censura o una licencia. Por otra parte, las numerosas correcciones que jalonan el texto están realizadas con una letra semejante a la del cuerpo, tan solo más pequeńa y menos inclinada, y con un trazo más delgado, para aprovechar el espacio disponible. El color de tinta es muy semejante al del resto, y solo a veces más oscuro.

Cabe indicar asimismo que el texto presenta en dos puntos tachaduras en diagonal con un claro intento censorio: el primero (Prosa Iv) por su carácter lascivo ( De lo que el luxurioso amante, poco curando, más aprieta consigo la bella nimpha, dispuesto de todo punto de llegar al fin su laçivo propósito", h. $17 \mathrm{v}$ ), el segundo (Prosa X), por referirse a un conjuro (h. 52r):

«Y entre estas cosas (assí como yo te enseñaré) atarás una ymagen de cera en tres ñudos con tres lazos de tres colores y tres vezes con ella en la mano andando al derredor del altar, otras tantas le punçarás el coraçón con punta de omicida espada, calladamente diziendo estas palabras:

Aquello hiero y ligo que trayo aquí comigo.

Tras esto tomarás alguna parte de la halda de su ropa y, plegándola poco a poco y assí plegada enterrándola en la cavada tierra, dirás:

Todo mi mal y mi enojo meto yo en este despojo.

Después, ençendiendo un ramo de verde laurel, añadirás:

Assí rechine en el fuego

quien mi mal se toma en juego.

En esto yo tomando una muy blanca paloma y tú quitándole una por una todas las plumas y hechándolas en el fuego, prosiguirás:

De la que me da el tormento

doy la carne y hueso al viento.

Al fin, después que toda la habrás despojado, dexándola sola yr, dirás assí el postrer encanto:

Quédate, malvada y cruda,

de la sperança desnuda.

Y cada vez que las sobredichas cosas harás, escupirás tres vezes, porque del número impar gozan los mágicos dioses.»

Estos signos de censura refuerzan la hipótesis de que la obra hubiera sido sometida a los controles preliminares para emprender su publicación. No sabemos por qué, pese a la aprobación de Ercilla, quedó inédito el poema $E l$ Victorioso Carlos Quinto y sepultado el manuscrito que lo contenía junto con la Arcadia tras pasar por el convento de Épila (este último fundado por los Urrea). No menos sombras planean sobre la fecha en la que nuestro autor tradujo la obra de Sannazaro. La pretensión de Geneste de situar este trabajo en los años cincuenta, ${ }^{6}$ choca con la ausencia total de alusiones a él en otras obras de Urrea,

6. Más concretamente, Geneste sitúa la traducción en la década 1550-1560, cf. Geneste (1978): 166-168. 
así como con el prefacio de Alfonso de Ulloa a su traslado al italiano del Diálogo de la verdadera honra militar (cf. Urrea 1569), donde, sin embargo, menciona la traducción del Furioso. Todo ello induce a considerarla como una labor tardía situable en años posteriores al regreso a España de Urrea tras su larga estancia en Italia, primero como capitán, luego como Virrey de Apulia. Recordaremos que, aun en la incertidumbre que rodea las fechas de su nacimiento y muerte esta última ha sido fijada con bastante probabilidad en torno a $1574 .{ }^{7}$

\section{La égloga XII y la traducción de Urrea}

Como es sabido, la redacción definitiva de la Arcadia, publicada en 1504, consta de una dedicatoria, un proemio y doce prosas, cada una seguida de una égloga; en fin, el ya mencionado epílogo $A$ la sampogna. ${ }^{8} \mathrm{El}$ proceso de composición había culminado hacia 1490, año de la muerte de la mujer de Pontano, cuya Égloga Meliseus constituye el precedente implícito de la XII de Sannazaro. ${ }^{9}$ Por ello Ricciardelli sostiene que «potrebbe chiamarsi l'epicedio dell'amore [...] un inno grandioso all'amore di due persone tolte dalla realtà» (Ricciardelli (1966): 119). En efecto, Meliseo representa aquí al propio Pontano que llora la pérdida de su esposa (Filis) y es a su vez llorado por Summonzio (Pietro Sommonte, futuro editor de la obra) y por Barcinio (Benedetto Gareth, Cariteo en la prosa II de la Arcadia). Una especie, pues, de llanto en abîme: el poeta llorado que llora a su vez a su amada esposa.

La égloga XII de la Arcadia, la más extensa de las que componen el prosímetro, gira, pues, en torno a la relación entre el ser humano, la naturaleza y la poesía, abriendo paso a un final esperanzado o, por lo menos, de aceptación resignada del destino. Ello ayuda a comprender la totalidad de la obra, si se entiende que la posibilidad de consuelo puede dirigirse a Sincero (Sannazaro), precisamente en el momento en que este, retornando a Nápoles tras su estancia en Arcadia, conoce la muerte de su propia amada. Ciertos atisbos de esperanza podían vislumbrarse ya en la prosa VIII, donde el personaje de Carino relata su dolorosa experiencia de desamor y recibe consuelo del amigo. Sin embargo, el final feliz de la obra queda desmentido por el epílogo, que entenebrece la perspectiva proyectando una visión de pérdida total: «Ogni cosa si perde, ogni speranza è mancata, ogni consolazione è morta» (Arcadia, 11). Según Antonio Gargano, de este modo, Sannazaro aplica la distinción de estilos y contenidos que

7. Para ulteriores detalles sobre este punto, remitimos a Geneste (1978) y a Cańas Gallart (2013).

8. Para la tardía inclusión de las prosas XI y XII junto con las respectivas églogas y el epílogo, así como para el incremento en la parte poética del influjo petrarquista y la mayor toscanidad de la lengua, véase Erspamer (1990): 29; el número entre paréntesis corresponde a la numeración en párrafos de esta edición.

9. Tateo considera la égloga XII como un «homenaje imitativo a Pontano», cf. Tateo (1993): 32. 
destinaba a la bucólica principalmente la temática del amor no correspondido o frustrado (Gargano (2005): 199). Entre ambos extremos se sitúa la égloga XII, que encierra las prerrogativas del amor arcádico, caracterizado, según Ricciardelli, por su vínculo con la Belleza, poseída a través del amor por la mujer, con la consiguiente implicación catastrófica que su muerte ocasiona para la armonía de la naturaleza y la vida pastoral (Ricciardelli, (1966:120).

En la Égloga XII, la naturaleza es el marco ineludible que comparte y difunde la tristeza del personaje, sugiriendo la unión entre lo mítico y lo real a través de lugares de la Campania y de Nápoles, ciudad biográficamente vinculada al autor y cuna, al mismo tiempo, de una larga tradición literaria. Una vez reconocida la importancia de la poesía en la expresión y la memoria del amor, los pastores inician un animado discurso acerca de sus efectos: hasta qué punto el canto poético puede ser consuelo para Meliseo, y puede también aliviar las cuitas de los pastores que lo escuchen. La relación entre naturaleza y poesía se materializa en el símbolo del laurel, leitmotiv que estructura la égloga, concebido como homenaje a la fuerza imperecedera del canto de Meliseo contra la muerte y el olvido, idea reforzada con las alusiones a personalidades literarias que dan prestigio a su poesía. El haya deviene punto de referencia espacio-temporal con que se abre y cierra la égloga; del mismo modo que árboles y aves son en numerosas ocasiones el canal de transmisión de los versos de Meliseo encargados de reiterar y amplificar su queja. Es además un paisaje dinamizado por la presencia del viento, que incluso adquiere relevancia como factor escénico al final del poema (v. 301), al actuar de obstáculo al acercamiento de Summonzio y Barcinio al lugar donde se encuentra Meliseo. Sannazaro consigue así dar un protagonismo esencial al paisaje bucólico que, en palabras de Muñiz, adquiere gracias a él una inédita función estructurante fundada en el poder difusor del canto poético. ${ }^{10}$ En la conclusión de la égloga, el verbo rinverdesi da entrada a dos fuerzas regeneradoras: por una parte, la de la esperanza y la renovación de la naturaleza que había quedado ensombrecida y estéril por la muerte de Filis; por otra, la continuidad del canto poético que se nutre de los sentimientos humanos, como fuerza creadora contrapuesta también a la muerte y al olvido.

El marco espacial de Nápoles incluye diferentes secuencias temporales. La primera se desarrolla en un tiempo presente con el diálogo bucólico entre Barcinio y Summonzio, al pie de un haya y otras plantas donde Meliseo ha escrito sus versos y lamentos. En la parte final de la Égloga, esta naturaleza es personificada y expresa también su lamento. El segundo episodio se refiere a la historia narrada a través de la voz de Barcinio en alusión a Meliseo y a los versos en que este relata un momento anterior en vida de Filis. Esta última analepsis adquiere connota-

10. «Il contributo più innovativo della bucolica sannazariana risiede... nell'aver costruito una sintassi complessa del paesaggio (con notevoli rispercussioni sulla sintassi della prosa), che fa ruotare il tutto attorno all'idea dell'accordo sinfonico", cf. Muñiz (2012): 138. 
ciones idílicas por la presencia de la mujer, en contraste con la desolación actual, que hace emanar de ese mismo espacio el dolor por la ausencia de la amada. Un desdoblamiento espacial entre pasado y presente, vida y fúnebre vacío, que Sannazaro resalta con toda evidencia. El teatro en que se hallan Barcinio y Summonzio, y en que discurre la acción narrada, podría describirse, pues, como un sistema de cajas chinas. Se trata, en suma, de un complejo cronotropo, cuyos círculos concéntricos se van llenando de resonancias a medida que cobra cada vez mayor protagonismo el espacio, hasta absorber el significado total de la historia. Este eje vertebra todos los planos narrativos, impregnando la subjetividad lírica y el ámbito dramático, como metáfora de la condición humana sometida al dictamen del tiempo, al cual solo sobreviven la naturaleza y sus ciclos.

Descendiendo al terreno de la métrica, la estructura y el estilo, la égloga, formada por 325 endecasílabos enlazados en tercetos encadenados con rima esdrújula (11A 11B 11A 11B 11C 11B 11C 11D 11C), se divide en tres partes. La primera (vv. 1-60), presenta como cronotropo un aquí-ahora ocupado por Barcinio y Summonzio que dialogan, y al que se volverá en la conclusión, consiguiendo así la estructura circular antes descrita y donde tienen cabida los sucesos protagonizados por Meliseo. La segunda parte (vv. 61-234) es la más extensa, y consta de varias secciones. El nudo de la acción narrada se centra en la expresión del lamento poético de Meliseo y en los efectos que este canto produce tanto en su corazón como en la naturaleza misma. Los diálogos entre Barcinio y Summonzio crean un movimiento escénico y dramático que ayuda a hilvanar los aspectos líricos, cuya acumulación acentúa gradualmente el patetismo en la expresión del dolor, hasta desembocar al final de la égloga en el impulso a visitar a Meliseo. La tercera parte (vv. 235-325) constituye la síntesis y depuración de los temas anteriores. Predomina la reflexión sobre la poesía y la memoria, con el añadido de nuevos matices: la esperanza de que la escritura y la naturaleza difundirán la fama de Filis y Meliseo, y la aceptación del destino, conforme al topos de la consolación. El lamento poético de Meliseo logra ahora el reconocimiento mediante la corona de laurel que hará perdurar su fama. El epitafio y el llanto de Meliseo ante Barcinio y Summonzio cierran la égloga situándonos de nuevo en el cronotropo inicial.

Los tercetos quedan delimitados por pausas sintácticas, aunque en algunos casos están enlazados para dar cabida a secuencias de distinta longitud, en ocasiones encaminadas al reforzamiento retórico. Predomina la esticomitia, si bien los encabalgamientos son asimismo significativos, en su mayoría acumulados en la segunda parte, donde se intensifica la expresión más vehemente del dolor. En cuanto al lenguaje, Sannazaro construye una sintaxis elaborada en la que abundan oraciones compuestas, tanto coordinadas como subordinadas, adjetivas en descripciones, y adverbiales para la expresión intensiva de los sentimientos. La égloga posee una rica adjetivación, muchas veces en forma de epítetos, así como numerosos verbos que confieren dinamismo a las situaciones. Estas palabras pueden presentarse en estructuras dobles parasinonímicas o incluso trimembres 
(v. 65: versi sol di dolor, lamenti e ritimi), con abundantes verbos creados por derivación (v. 105, inolmi o impopuli), presencia de latinismos (v. 100, diverticuli; v. 101, scopuli) y léxico culto, como segundo componente de la duplicación. En el nivel fonético, algunas aliteraciones refuerzan el motivo de una naturaleza sensorial y dinámica, transmisora de sonidos significativos que confieren densidad canora al lenguaje. Por otra parte, dos onomatopeyas (v. 201 y 223) y la personificación de las aves sugieren una concepción de la naturaleza como resonancia del lamento y los versos pastorales, motivo recurrente en la Arcadia. ${ }^{11}$

El análisis aquí realizado da la medida de las dificultades con las que los traductores hubieron de enfrentarse para respetar el juego de antítesis, correspondencias, alusiones y juegos temporales que esta égloga contiene. A continuación, para poder asignar a la versión urreiana de la Arcadia el valor que le corresponde, analizaremos algunos casos concretos comparando sus soluciones con las ofrecidas por los otros traductores quinientistas.

A diferencia de Salazar, tanto Urrea como Sedeño y Viana respetaron, aunque en diversa medida, la métrica original. Aparte de este dato, si comparamos en su conjunto las cuatro versiones, constatamos que Salazar adaptó el texto al ámbito cultural castellano ya sea modificando el sentido para introducir rasgos más reconocibles, ya sea suprimiendo algunos elementos del mundo mítico o bucólico poco familiares para el lector español. Esa misma razón lo impulsó a subrayar los rasgos teatrales y dramáticos del texto más acordes con la tradición del diálogo entre pastores. Como hemos dicho, la mayoría de las correcciones posteriores de Garay estuvo encaminada a restaurar el sentido original en el nivel léxico; en otros casos su fin fue clarificar algunas estructuras sintácticas que podían dificultar la lectura. La edición de 1547 recuperaba así algunos aspectos característicos de la bucólica culta y del mundo arcádico sannazariano, aunque sin remediar la desarticulación de su refinado juego de antítesis, correspondencias, superposiciones y desdoblamientos temporales.

En cuanto a la versión de Jerónimo de Urrea, no solo respeta la métrica original, sino que lo hace sistemáticamente, incluso en lo que concierne a los tercetos encadenados. La adopción de medidas semejantes a las empleadas por Sannazaro, le permite adaptarse mejor a los matices retóricos y sintácticos del original. Por lo demás, lejos de incurrir en un calco mecánico, se sirve de leves trasposiciones que incrementan la naturalidad del resultado en castellano. Veamos algunos ejemplos.

11. Para un estudio de la relación dinámica entre poesía y naturaleza, remito a Muñiz (2012): $133-161$. 


\begin{tabular}{|l|l|}
\hline Sannazaro (vv. 4-6) & $\begin{array}{l}\text { Oh pietà grande! E quali dii permisero } \\
\text { a Meliseo venir fato tant'aspero? } \\
\text { Perché di vita pria non lo divisero? }\end{array}$ \\
\hline Salazar, ed. de 1547 & $\begin{array}{l}\text { Su. ¡Oh piedad fallescida! } \\
\text { ¿Quáles dioses permitieron } \\
\text { a Meliseo tal cayda? } \\
\text { ¿Por qué de vida primero } \\
\text { su cuerpo no dividieron? }\end{array}$ \\
\hline Urrea & $\begin{array}{l}\text { ¿Oh gran piedad, oh dios que permitiste } \\
\text { que huviesse en Meliseo tal aspereça! } \\
\text { ¿Por qué el hilo vital no le rompiste? }\end{array}$ \\
\hline
\end{tabular}

Como vemos, al traducir el verso "Perché di vita pria non lo divisero?» como "¿Por qué el hilo vital no le rompiste?», Urrea recurre a una metáfora que le permite preservar la fluencia y el ritmo de la cláusula. Este resultado melódico contrasta con la fragmentación del verso y su sometimiento a un ritmo sincopado en Salazar-Garay: «de vida primero / su cuerpo no dividieron». En cuanto al sintagma fato tantaspero del verso anterior, queda felizmente resuelto en Urrea por la sustantivación abstracta que lo reduce a cualidad («tal aspereça»), frente a la prolija perífrasis del predecesor.

Por otra parte, si la versión de Salazar-Garay traduce de forma más literal el léxico y mantiene en mayor grado las estructuras sintácticas, pese a los rellenos y desviaciones impuestos por el distinto metro, Urrea es más flexible con la letra precisamente para salvaguardar la estructura métrica del original. Pero no se trata de libertades arbitrarias, sino de equivalencias encaminadas a salvar obstáculos incompatibles con logros retórico-estilísticos considerados esenciales. Hay, en cualquier caso, una estrategia selectiva alejada del método "palabra por palabra» al que en última instancia responde la técnica de Salazar.

Si ampliamos la comparación a las otras dos traducciones inéditas, comprobamos que también estas emplean el endecasílabo evitando por lo general, como Urrea, los finales esdrújulos, aunque este último es quien se ciñe de forma más sistemática al endecasílabo llano, ya impuesto por Garcilaso como signo de elegante modernidad renacentista. En cuanto al número de estrofas, Urrea sigue estrictamente la división del original, mientras que Viana añade cuatro tercetos y Sedeño, doce. En el tratamiento de la rima, es sabida la propensión de Sannazaro por las derivadas (aspero, esaspero, inaspero), desinenciales (piangere, tangere, frangere), y equívocas (specoli, specoli) (Erspamer 1990: 224). Por otra parte, Urrea y Viana tienden a conservar las categorías gramaticales del original, mientras que Sedeño las modifica e incluso acude a palabras diferentes. Aunque todos recurren a veces al hipérbaton para resolver problemas métricos, Urrea evita abusar de esta figura para no forzar la sintaxis; sin embargo, la mantiene cuando en el original cumple una función expresiva importante. Es el caso de la solución dada a una prótasis intercalada como inciso para resaltar 
el hecho extraordinario de que Meliseo (que habla en primera persona) siga vivo pese a tanto dolor: "Onde con questo mio dir..., sio vivo, ancor farò...". Viana cancela este efecto expresivo posponiendo la prótasis, mientras que Sedeño elimina incluso la construcción hipotáctica. Frente a estas dos soluciones empobrecedoras, la de Urrea es, sin duda, la más elegante y adecuada, no solo porque mantiene el orden del período condicional, sino porque logra también reproducir el inciso:

\begin{tabular}{|l|l|}
\hline Sannazaro (vv. 256 - 258) & $\begin{array}{l}\text { Onde con questo mio dir non incelebre, } \\
\text { sंio vivo, ancor farò tra questi rustici } \\
\text { la sepoltura tua famosa e celebre. }\end{array}$ \\
\hline Urrea & $\begin{array}{l}\text { Do con mi canto no desnudo de arte } \\
\text { espero, si yo vivo, entre silvanos } \\
\text { celebrar tu sepulcro en esta parte. }\end{array}$ \\
\hline Sedeño & $\begin{array}{l}\text { Yo haré que tu nombre se çelebre, } \\
\text { y en estos bosques sea y estos llanos } \\
\text { famoso tu sepulcro y muy çélebre. }\end{array}$ \\
\hline Viana & $\begin{array}{l}\text { Por eso, te honraré perpetuamente, } \\
\text { si vivo, y aun haré de buena gana } \\
\text { famoso tu sepulcro y excellente. }\end{array}$ \\
\hline
\end{tabular}

Algunos cambios léxicos y semánticos se imbrican con los ya descritos. Así, el añadido de palabras para explicitar significados implícitos en el original, amplifica la parte descriptiva ralentizando el ritmo. Urrea — que tiende a añadir sobre todo adjetivos- es, también en este caso, quien consigue efectos estilísticos más logrados. Veamos algunos ejemplos: el verso 157 contiene una oración exclamativa, donde la dictología parasinonímica (pianto e gemito) acentúa el dolor de Meliseo: «Oh lasso, oh dí miei volti in pianto e gemito!». Aquí Urrea elimina la dictología pero compensa la supresión añadiendo la antinomia adjetival «blando»/duro» para referirse respectivamente al pasado y presente. De este modo la antítesis implícita entre los "dí» felices convertidos (volti) en llanto, recibe mayor evidencia: « $\mathrm{Oh}$ blando tiempo vuelto en duro llanto!». En el verso 49, el adjetivo añadido aporta una cualidad sonora connotada que subraya aún más la desolación: "Non gían con un suon tristo e miserabile» > "Con un son triste, ronco y miserable». Mientras que en el v. 33 Urrea añade un sintagma que explicita el elemento del paisaje solo aludido por Sannazaro: «e con un salto poi ti apprendi e sbàlzati» > "salta y en la alta cumbre d'él te enxalça».

La superioridad del método de Urrea respecto al de los otros dos traductores se aprecia también en los vv. 211-213, donde Sannazaro sitúa como palabras rima la seudo antítesis obtenebre / distenebre, aludiendo la primera al cielo, la segunda al ánimo de Meliseo, ambos igualmente oscurecidos por la pérdida del «sol» que los iluminaba: 
Ovunque miro par che'l ciel si obtenebre, ché quel mio sol che l'altro mondo allumina è or cagion ch'io mai non mi distenebre.

Urrea sortea la dificultad sustituyendo los verbos por sendas perífrasis metafóricas, pero manteniendo su sentido y su posición en final de verso. Por contra, los otros dos traductores recurren a paráfrasis totalmente alejadas de la letra, la sintaxis e incluso el significado de los versos, si bien Sedeño logra una imitación meramente fónica de las palabras-rima (pesadumbre / alumbre):

\begin{tabular}{|l|l|}
\hline Urrea & $\begin{array}{l}\text { El cielo veo con escuro manto, } \\
\text { que aquel mi sol que al otro mundo aclara } \\
\text { causa que viva ciego en dolor tanto. }\end{array}$ \\
\hline Sedeño & $\begin{array}{l}\text { Tu claridad doquiera es pesadumbre, } \\
\text { que después que mi sol alumbra el çielo, } \\
\text { no ay cosa que me alegre, ni me alumbre. }\end{array}$ \\
\hline Viana & $\begin{array}{l}\text { Aquel mi sol por quien viviendo muero } \\
\text { es causa que a do miro se escuresce, } \\
\text { Desque de es' otro mundo fue luzero. }\end{array}$ \\
\hline
\end{tabular}

Operaciones de amplificación, reducción y sustitución se perciben ciertamente en todos los traductores, obligados a medirse con dificultades a menudo insuperables. Sin embargo, mientras que Sedeño y Viana oscilan a menudo entre dos extremos: la traducción literal y la excesiva libertad, Urrea mantiene una singular coherencia y un mayor equilibrio, en cuya base está una estrategia más refinada y meditada de traducción.

Esta técnica se manifiesta asimismo en el moderado recurso a figuras retóricas ausentes en el original que, en cualquier caso, no alteran el sentido del texto. Excepcionales son, de hecho, las intromisiones dictadas por motivos ideológicos, mientras que es bastante sistemática la reducción de latinismos, a menudo traducidos con formas patrimoniales del léxico castellano. Es significativo también notar que las variantes por él introducidas al corregir la versión, buscan aproximarse más a la letra original, modernizar la lengua y ennoblecerla, rasgos estos dos últimos muy presentes también en los retoques aportados a la versión ayaliana de las prosas. Todo ello confirma la impresión de hallarnos ante una obra de madurez, la misma que se refleja en El Victorioso Carlos Quinto, donde el lenguaje utilizado responde a un equilibrio renacentista bien reconocible.

Esperamos que el conocimiento de esta última empresa de Urrea como traductor sirva para desterrar el prejuicio difundido por Cervantes. A la imagen estática y casi proverbial de 'mal traductor', debería contraponerse, pues, la de un literato exigente consigo mismo, capaz de buscar difíciles equilibrios entre fidelidad a la forma y a la letra, atento al estilo del autor original y enri- 
quecido por anteriores experiencias. Por todo ello, el ms. 1469 de la Biblioteca Nacional constituye un documento de primer orden no solo para conocer cabalmente la personalidad literaria de Urrea, sino también para reconstruir un eslabón perdido de la cadena de esfuerzos encaminados a enriquecer la lengua castellana como lengua de traducción poética a lo largo del siglo xvi. El esmero y mejora de su método, ulteriormente atestiguado por las variantes introducidas en el proceso de copia, evidencian además su interés en publicar la traducción, hipótesis reforzada por los indicios que aportan el paratexto y el estudio de la grafía.

Desconocemos quién decidió iniciar los trámites de impresión y por qué luego esta no se llevó a fin, pero creemos haber probado que la traducción de la Arcadia, al igual que el poema que la acompañaba en el manuscrito, El Victorioso Carlos Quinto, no fueron fruto de un trabajo provisional y desechado, sino corregido hasta el final y destinado a imprimirse.

Publicamos, pues, la traducción de la égloga XII con al frente el original sannazariano, este último conforme a la edición de Erspamer.

\section{Criterios de edición}

La transcripción del texto es conservativa, a fin de ofrecer en su autenticidad la lengua del traductor en una época de notables oscilaciones y dinamismo evolutivo del castellano. Para ello, se han adoptado los siguientes criterios:

- Se normaliza la oposición u/v según el criterio actual.

- Se adopta la norma actual en lo que concierne a la consonante $r$.

- Se escribe $m$ antes de $b$ y $p$.

- La grafía $\int$ se transcribe como $s$.

- Se añade $h$ para distinguir la interjección $o h$ de la conjunción disyuntiva $o$.

- Se resuelven las abreviaturas sin señalarlas entre corchetes para favorecer la fluencia del texto.

- Se introduce la acentuación y la puntuación modernas para favorecer la inteligencia del texto.

- Se regulariza la separación de palabras y se marca con apóstrofe la elipsis de vocales.

- En los versos, se marca la diéresis con el signo tipográfico correspondiente.

- Por contra, se respetan los paréntesis y las oscilaciones ortográficas.

- Se respetan asimismo las oscilaciones del texto en lo que atañe a mayúsculas y minúsculas, salvo en el caso de nombres propios o palabras precedidas de punto. A comienzo de verso se conserva la mayúscula allí donde aparece en el ms.

- Los errores, u omisiones debidos a causas diversas, se restituyen con los signos $<>$ y se anota a pie de página la lectura enmendada o los casos inciertos.

- Las palabras o segmentos problemáticos, no solucionables mediante conjetura, se enmarcan con el signo + 
La edición crítica da cuenta, en fin, de todas las correcciones y variantes. Tras el signo ] se transcriben las variantes eliminadas, utilizando el símbolo tipográfico $><$ para tachado. Las aclaraciones sobre el sentido del texto, así como las versiones de Sannazaro y Ayala/Garay se consignan en nota a pie de página, utilizando las siguientes abreviaturas:

corr. inter:: corrección interlineal.

sobreescr.: variante sobreescrita sobre otra tachada.

Por último, en nuestros comentarios a pie de página, $S$ se refiere al texto de Sannazaro, y U, al de Urrea. 


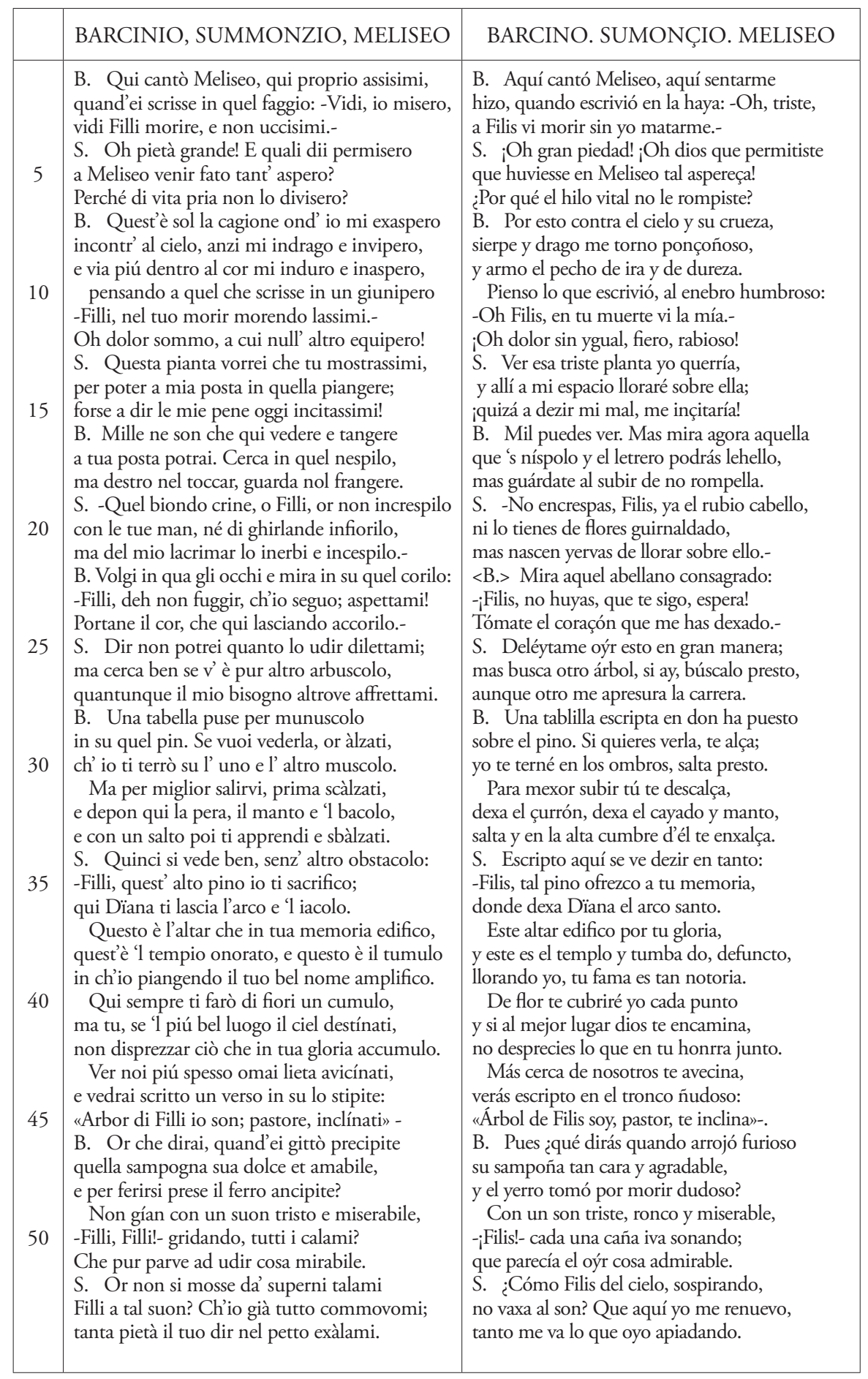


55 B. Taci, mentre fra me ripenso, e provomi se quell'altre sue rime or mi ricordano, de le quali il principio sol ritrovomi. S. Tanto i miei sensi al tuo parlar si ingordano, che temprar non gli so. Comincia, agiútati; ché ai primi versi poi gli altri s’accordano. B. -Che farai, Meliseo? Morte refútati, poi che Filli t'ha posto in doglia e lacrime, né piú, come solea, lieta salútati.

Dunque, amici pastor, ciascun consacrime versi sol di dolor, lamenti e ritimi; e chi altro non può, meco collacrime.

A pianger col suo pianto ognuno incitimi, ognun la pena sua meco communiche, benché 'l mio duol da sé dí e notte invitimi.

Scrissi i miei versi in su le poma puniche, e ratto diventâr sorba e corbezzoli; sí son le sorti mie mostrose et uniche.

E se per inestar li incido o spezzoli, mandan sugo di fuor sí tinto e livido, che mostran ben che nel mio amaro avezzoli.

Le rose non han piú quel color vivido, poi che 'l mio sol nascose i raggi lucidi dai quai per tanto spazio oggi mi dívido.

Mostransi l'erbe e i fior languidi e mucidi, i pesci per li fiumi infermi e sontici, e gli animai nei boschi incolti e sucidi.

Vegna Vesevo, e i suoi dolor racontici. Vedrem se le sue viti si lambruscano e se son li suoi frutti amari e pontici.

Vedrem poi che di nubi ognor si offuscano le spalle sue, con l'uno e l'altro vertice; forse pur novi incendî in lui coruscano.

Ma chi verrà che de' tuoi danni accertice Mergilina gentil, che sí ti inceneri, e i lauri tuoi son secche e nude pertice?

Antinïana, e tu perché degeneri? Perché ruschi pungenti in te diventano quei mirti che fur già sí molli e teneri?

Dimmi, Nisida mia (cosí non sentano le rive tue giamai crucciata Dorida, né Pausilipo in te venir consentano!), non ti vid'io poc' anzi erbosa e florida, abitata da lepri e da cuniculi? Non ti veggi'or piú ch'altra incolta et orida?

100 Non veggio i tuoi recessi e i diverticuli tutti cangiati, e freddi quelli scopuli dove temprava Amor suo' ardenti spiculi?

Quanti pastor, Sebeto, e quanti populi morir vedrai di quei che in te s'annidano, pria che la riva tua si inolmi o impopuli? Lasso, già ti onorava il grande Erídano, e 'l Tebro al nome tuo lieto inchinavasi; or le tue ninfe appena in te si fidano.

Morta è colei che al tuo bel fonte ornavasi,
B. Calla, mientra que pienso un poco, y pruevo si aquellos otros versos se me acuerdan, que no holvidé el principio estraño y nuevo. S. De pura compasión se desacuerdan mis sentidos. Con esso, tú te ayuda, que tras el primer verso otros se acuerdan. B. -¿Qué harás, Meliseo? Que muerte duda de ti, pues te dio Filis tal castigo, ni qual solía alegre te saluda.

Conságreme el pastor caro y amigo versos con gran dolor y descontento; y quien no puede más, llore comigo.

Incíteme a llorar su sentimiento, comigo traten todos mis cuydados, puesto que por sí duele mi tormento.

Mis versos escriví por los granados, y servales azedos se volbieron; así son monstrüosos ya mis hados.

Los que por enxerillos se rompieron, hecharon fuera lágrimas moradas, creo que en mi amargura se tiñeron.

Ya se muestran las rosas desmayadas, después que aquel mi sol no les da vida, lexos de quien estoy tantas jornadas.

La yerva y flor está mustia y caýda, los peces por los ríos van dolientes, la fiera por las sierras desabrida.

Diga Vesubio aquí sus acidentes, si sus parras en cierne reverdecen, si amargos son sus fructos diferentes.

Veremos si de nuves se escurecen sus espaldas verdíssimas, y alturas; o si por nuevos fuegos resplandecen.

¿Quién vendrá a reparar tus desventuras, que heres ceniça ya, oh Mergelina, y tus laureles varas secas, duras?

¿Tu fuerça, Antiniana, ya declina? ¿Por qué espinos se tornan y no aumentan en ti aquel verde mirto y rosa fina?

Dime, Nisida mía, jasí no se sientan tu ribera la Dorida salada, ni Pausilipo a ti venir consientan!

¿No te vi yo de flores coronada, de liebres y conejos abundosa? ¿No estás más que otras seca, despoblada?

No veo tu cañada tan hermosa trocada, veo y la fragua fría, ardiente, do templava el amor flecha amorosa?

¿Quánto pastor, Sebeto, aquí excelente, verás morir y pueblo, antes del día que pueblen olmo y álamo tu fuente?

Honrrarte el grand Erídano solía, el Tíber a tu nombre se inclinava; ora, apenas tu Nimpha en ti se fía.

Ya es muerta quien tu fuente guirnaldava, siendo espejo de todos tu hondura; 
onde tua fama al ciel volando alzavasi.

Or vedrai ben passar stagioni e secoli, e cangiar rastri, stive, aratri e capoli, pria che mai sí bel volto in te si specoli.

Dunque, miser, perché non rompi e scapoli tutte l'onde in un punto et inabissiti, poi che Napoli tua non è piú Napoli?

Questo dolore, ohimè, pur non predissiti quel giorno, o patria mia, ch'allegro et ilare tante lode, cantando, in carta scrissiti.

Or vo' che 'l senta pur Vulturno e Silare, ch'oggi sarà fornita la mia fabula, né cosa verrà mai che 'l cor mi exílare.

Né vedrò mai per boschi sasso o tabula ch'io non vi scriva "Filli», acciò che piangane qualunque altro pastor vi pasce o stabula.

$E$ se adverrà che alcun che zappe o màngane da qualche fratta, ov'io languisca, ascoltemi, dolente e stupefatto al fin rimangane.

130 Ma pur convien che a voi spesso rivoltemi, luoghi un tempo al mio cor soavi e lepidi, poi che non trovo ove piangendo occoltemi.

O Cuma, o Baia, o fonti ameni e tepidi, or non fia mai che alcun vi lodi o nomini, che 'l mio cor di dolor non sude e trepidi.

E poi che morte vuol che vita abomini, quasi vacca che piange la sua vitula andrò noiando il ciel, la terra e gli uomini.

Non vedrò mai Lucrino, Averno o Tritula, che con sospir non corra a quella ascondita valle che dal mio sogno ancor si intitula.

Forse qualche bella orma ivi recondita lasciâr quei santi piè, quando fermarosi al suon de la mia voce aspra et incondita; e forse i fior che lieti allor mostrarosi faran gir li miei sensi infiati e tumidi de l'alta vision ch' ivi sognarosi.

Ma come vedrò voi, ardenti e fumidi monti, dove Vulcan bollendo insolfasi,

150 che gli occhi miei non sian bagnati et umidi?

Però che ove quell' acqua irata ingolfasi, ove piú rutta al ciel la gran voragine e piú grave lo odor redunda et olfasi, veder mi par la mia celeste imagine sedersi, e con diletto in quel gran fremito tener le orecchie intente a le mie pagine.

Oh lasso, oh dí miei volti in pianto e gemito! Dove viva la amai, morta sospirola, e per quell'orme ancor m'indrizzo e insemito. Il giorno sol fra me contemplo e mirola, e la notte la chiamo a gridi altissimi; tal che sovente infin qua giú ritirola.

Sovente il dardo, ond'io stesso trafissimi, mi mostra in sogno entro i begli occhi, e dicemi:
"Ecco il rimedio di tuoi pianti asprissimi».

E mentre star con lei piangendo licemi, donde tu fama hasta el cielo alçava.

Siglo verás pasar y hedad madura, trocar reja y açada y primavera, antes que en ti se espeje su hermosura.

¿Cómo no quiebras, cómo no hechas fuera de ti tus hondas?, di, ¿qué estás dudando, pues Nápoles no es Nápoles qual hera?

Ay que yo fuy tu mal adevinando, oh dulze patria, el día que contento tanto loor scriví en papel, cantando.

Volturno y Silar sientan lo que cuento, que oy mi fábula cumplo y quedo en guerra, donde paso la vida en gran tormento.

No veré tabla o piedra en monte o sierra donde no escriva "Filis», porque llore qualquier pastor que hervase en esta tierra.

Y si acaheze ${ }^{12}$ que alguno siembre o more aquí, oya mi voz, porque no dudo que en ansias y tristeças empeore.

Mas conviene que a vos torne a menudo, lugar que un tiempo fuysteys y aplaciente, pues no hallo quien oya mi mal crudo.

¡Oh Cuma, oh Vaya, oh clara y suave fuente!, ¿quién hos podrá nombrar en esta sierra, que mi pecho no sude amargamente?

Pues tengo con la vida amarga guerra, qual vaca por la hija yré bramando, enojando los cielos y la tierra.

Nunca Averno y Locrino yré mirando, que no corra con llanto al escondido valle que aún de mi sueño van nombrando.

Quizá podré hallar rastro imprimido de aquellos sanctos pies, que allí pararon al son d'esta mi voz ronca y gemido; quizá flores que alegres se mostraron harán a mis sentidos presuntuosos de aquella alta visión que allí soñaron.

¿Mas cómo hos veré, ardientes y humosos montes, do vive en la açúfera vena Vulcán, sin ver mis ojos muy llorosos?

Que do se engolfa más su agua y suena, y donde al çielo más su llama invía, donde es la olor más grave y da más pena,

Allí veo la celeste ymagen mía sentada, y con plazer oýr mi canto y estar atenta al son y a la armonía.

¡Oh blando tiempo vuelto ${ }^{13}$ en duro llanto! Donde viva la amé y muerta sospiro, y sigo el rastro que seguí ya tanto.

El día entre mí yo la contemplo y miro, y la noche, con grito alto y gemido, muchas vezes de allá a mí la retiro.

Durmiendo, el dardo, con que fuy herido, en sus ojos me muestra y va diziendo: «He aquí el remedio de tu mal creçido».

Mientra con ella puedo estar gimiendo, 


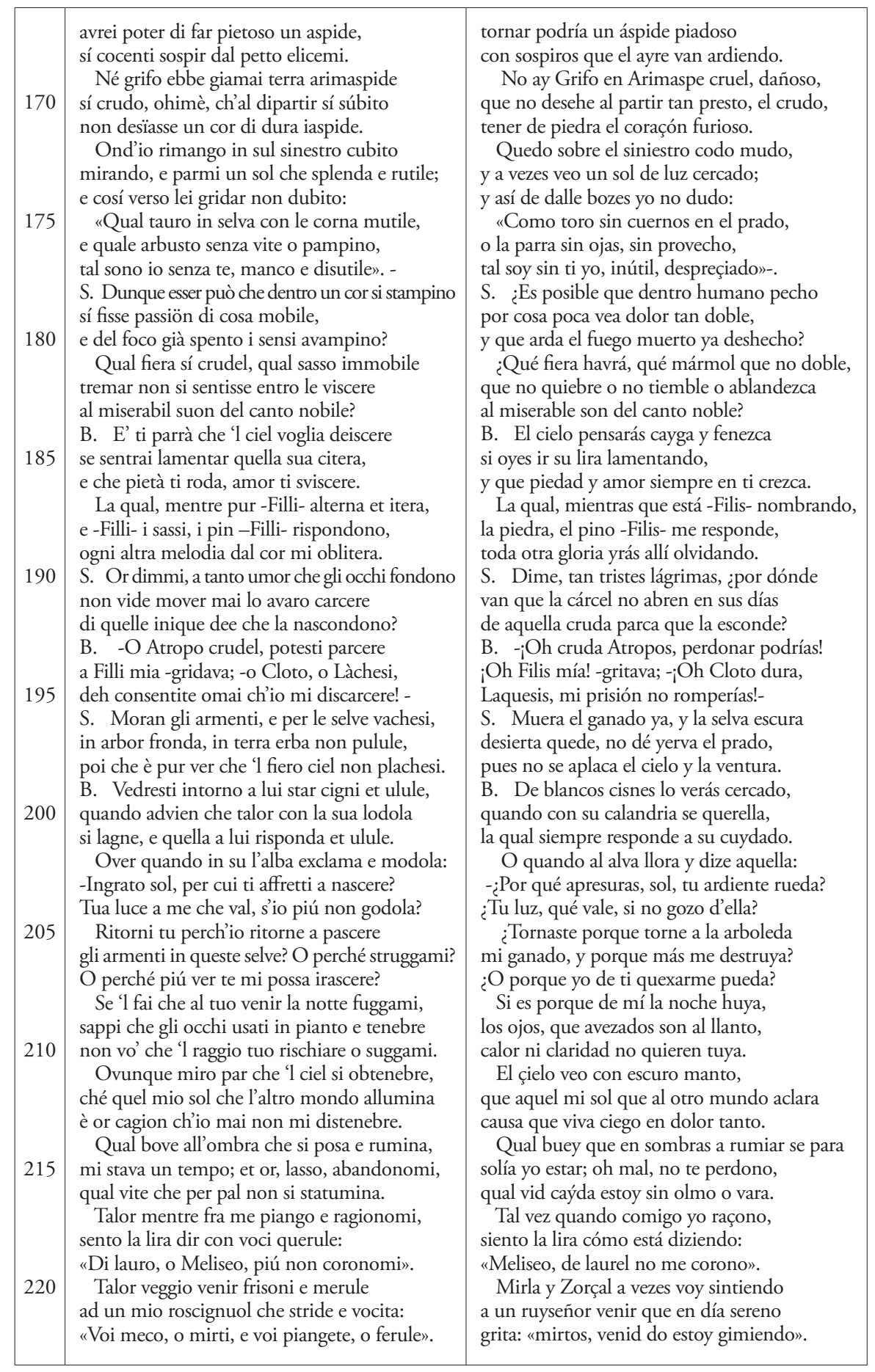


Talor d'un' alta rupe il corbo crocita: «Absorbere a tal duolo il mar devrebbesi Ischia, Capre, Ateneo, Miseno e Procita».

La tortorella, che al tuo grembo crebbesi, poi mi si mostra, o Filli, sopra un àlvano secco, ché in verde già non poserebbesi; e dice: «Ecco che i monti già si incalvano,

230 o vacche, ecco le nevi e i tempi nubili; qual'ombre o qua' difese omai vi salvano?».

Chi fia che, udendo ciò, mai rida o giubili? E' par che i tori a me, muggendo, dicano: «Tu sei, che con sospir quest' aria annubili». -

235 S. Con gran ragion le genti s'affaticano per veder Meliseo, poi che i suoi cantici son tai che ancor nei sassi amor nutricano.

B. Ben sai tu, faggio che coi rami ammantici, quante fiate a' suoi sospir movendoti

240 ti parve di sentir suffioni o mantici.

O Meliseo, la notte e 'l giorno intendoti, e sí fissi mi stan gli accenti e i sibili nel petto, che, tacendo ancor, comprendoti. S. Deh, se ti cal di me, Barcinio, scribili, 245 a tal che poi, mirando in questi cortici, l'un arbor per pietà con l'altro assibili.

Fa che del vento il mormorar confortici, fa che si spandan le parole e i numeri, tal che ne sone ancor Resina e Portici.

250 B. Un lauro gli vid'io portar su gli umeri, e dir: -Col bel sepolcro, o lauro, abbràcciati, mentre io semino qui menta e cucumeri.

Il cielo, o diva mia, non vuol ch'io tàcciati, anzi, perché ognor piú ti onori e celebre, dal fondo del mio cor mai non discàcciati.

Onde con questo mio dir non incelebre, s'io vivo, ancor farò tra questi rustici la sepoltura tua famosa e celebre.

E da' monti toscani e da' ligustici

260 verran pastori a venerar quest' angulo, sol per cagion che alcuna volta fustici.

E leggeran nel bel sasso quadrangulo il titol che a tutt'ore il cor m'infrigida, per cui tanto dolor nel petto strangulo:

265 "Quella che a Meliseo sí altera e rigida si mostrò sempre, or mansüeta et umile si sta sepolta in questa pietra frigida».-

S. Se queste rime troppo dir presumile, Barcinio mio, tra queste basse pergole,

270 ben veggio che col fiato un giorno allumile. B. Summonzio, io per li tronchi scrivo e vergole, e perché la lor fama piú dilatesi, per longinqui paesi ancor dispergole; tal che farò che 'l gran Tesino et Atesi,

275 udendo Meliseo, per modo il cantino, che Filli il senta et a se stessa aggratesi; e che i pastor di Mincio poi gli piantino un bel lauro in memoria del suo scrivere,
Veo el cuervo graznar de dolor lleno: «Tragarse el mar a Proxita devría, a Iscla, a Capri, a Ateneo y a Miseno». La Tórtola, que en tu halda vivía, oh Filis, en un seco olmo aparece, que en árbol verde ya no posaría; y dize: «Assí la sierra se encalveçe, vacas, la nieve al mundo aya ocupado; ¿qué sombra o qué reparo os favoreçe?»

¿De qué reyrá quien esto ha contemplado? Bramando el toro dize claramente: "Tu sospirar nos ha el tiempo anublado»-. S. Con gran raçón desea ver la gente a Meliseo, pues canta versos tales, que en piedras pinta amor eternamente. B. Quando escuchavas, haya, sus caudales sospiros, que aún temblar d'ellos te veo, fuelles sentías alçados de sus males.

La noche y día te oyo, oh Meliseo, y en mi coraçón pongo tus raçones, $y$, aquí callando, entiendo tu deseo.

S. Barcino, escrive, escrive sus cançiones, porque en estas corteças remirando, silve un árbol con otro tristes sones.

El viento al murmurar vaya esforçando, tiéndanse las querellas que yo nombro, por Porto y por Reasina resonando.

B. Vile traher un lauro sobre el hombro, diziendo: -Abraça tú la sepoltura, mientras que siembro aquí menta y cohombro.

No quiere, diosa mía, mi ventura que calle, antes, por más gran fama darte, jamás saldrá del alma tu figura.

Do con mi canto no desnudo de arte espero, si yo vivo, entre silvanos celebrar tu sepulcro en esta parte.

Que de montes ligustros a toscanos, porque fuyste en el mundo solamente, vengan tu pira a ver moços y ancianos.

Leherán en esse mármol eminente el título que el coraçón me enfría, por quien tanto dolor mi alma siente:

«La que soberbia y dura cada día fue a Meliseo, aquí en eterno asiento se encierra humilde en esta piedra fría».

S. Si cantas muchas vezes lo que cuento entre las baxas parras d'estas tierras, un día se encenderán del solo aliento. B. Escrívolos por troncos d'estas sierras, que a ello a mí su fama persüade, y espárçolos por muy remotas tierras;

Tal que haré que el gran Tesín y el Ade, oyendo Meliseo, de un arte canten, que lo oya Filis y assí propia agrade;

$Y$ pastores de Minçio $<a>^{14}$ aqueste planten en su memoria un lauro consagrado, 


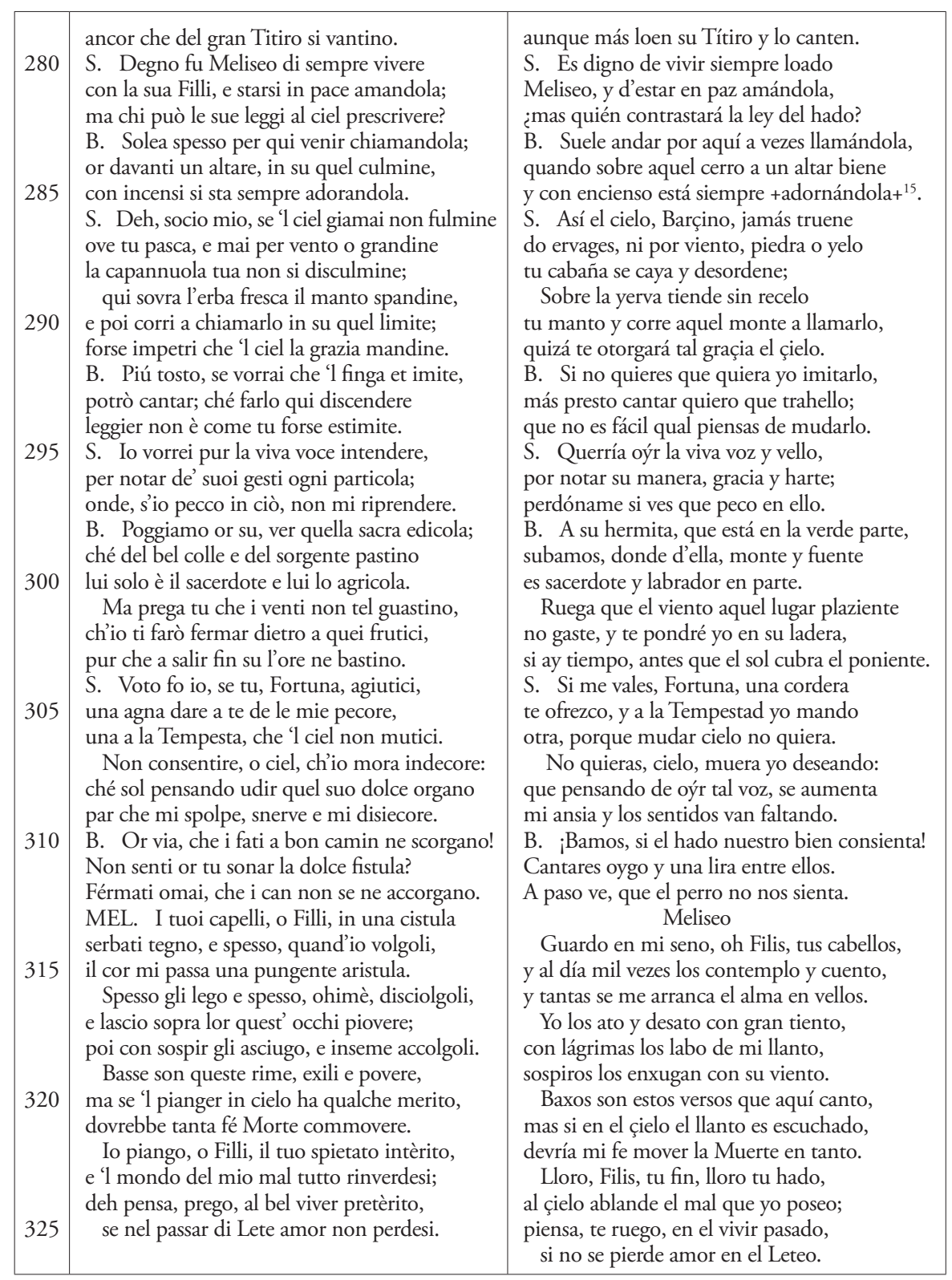

12. acaheze, corr. inter.] >avendrá <

13. vuelto, sobreescr.] $>\mathrm{b}<$ uelto

14. a aqueste, en el ms. aqueste, pero no es acusativo, sino dativo, cfr., S.: gli piantino.

15. adornándola. Así en el ms., pero cfr. S.: adorandola. Ante la duda de si es error de lectura o de copia, hemos optado por no intervenir con una enmienda. 


\section{Bibliografía}

Ariosto, Ludovico, Orlando furioso, edición bilingüe de la traducción de Jerónimo de Urrea (1549), a cargo de Cesare Segre y M. de las Nieves Muñiz Muñiz, Madrid, Cátedra, 2002.

Borao, Jerónimo, Noticia de D. Gerónimo Jiménez de Urrea y de su novela caballeresca inédita D. Clarisel de las Flores, Zaragoza [s.n.], Imprenta Calisto Ariño, 1866, pp. 7-56

CaÑas Gallart, Cecilia, La traducción de la "Arcadia» de Sannazaro por Jerónimo Jiménez de Urrea (s. XVI). Estudio y edición crítica, Universitat de Barcelona, 2013, URL: http://www.tdx.cat/handle/10803/111043

Erspamer, Francesco (ed.), Iacopo Sannazaro, Arcadia, Milán, Mursia, 1990.

Gargano, Antonio, «Da Sannazaro a Garcilaso: traduzione e transcodificazione (a proposito della seconda egloga)», en Primo Convengo Internazionale sulla traduzione della letteratura italiana in Spagna (1300-1939), ed. de María de las Nieves Muñiz Muñiz, con la colaboración de Laura Calvo Valdivieso y Ursula Bedogni, Florencia, Franco Cesati, 2007.

—, «Legloga a Napoli tra Sannazaro e Garcilaso», en Id. Con accordato canto, Napoli, Liguori Editore, 2005.

Geneste, Pierre, Le capitaine-poète aragonais Jerónimo de Urrea, sa vie et son œuvre ou Chevalerie et Renaissance dans l'Espagne du XVI siècle, Paris, Ediciones hispanoamericanas 26, 1978, p. 208.

LAPESA, Rafael, La trayectoria poética de Garcilaso, 2a edición, Madrid, Alianza Editorial, 1968.

Morros, Bienvenido, véase Vega, Garcilaso de la.

Muñiz MuñIz, María de las Nieves, Fonti grecolatine e locus amoenus': tra Sannazaro e Garcilaso, en Id., L'immagine riflessa. Percezione nazionale e trame intertestuali fra Italia e Spagna, Florencia, Franco Cesati, 2012.

Reyes Cano, Rogelio, La Arcadia de Sannazaro en España, Sevilla, Anales de la Universidad Hispalense, serie de Filosofía y Letras, núm. 16, 1973.

Ricciardelli, Michele, L’Arcadia di J. Sannazaro e di Lope de Vega, Napoli, Fausto Fiorentino Editore, 1966.

TAteo, Francesco, «La Arcadia en España», en Jacopo Sannazaro, Arcadia, Madrid, Cátedra, 1993.

URREA, Jerónimo Jiménez de, Dialogo del vero honore militare, nel quale si diffniscono tutte le querele, che possono occorrere fra l'uno e l'altr'buomo. Con molti notabili esempii d'antichi, \& moderni. Composto dallillustre sig. Don Geronimo di Vrrea... Et nuouamente tradotto di lingua spagnuola da Alfonso Vlloa, in Venetia, appresso gli heredi di Marchio Sessa, 1569.

Vega, Garcilaso de la, Obra poética y textos en prosa, Barcelona, Crítica, 2001. 